\title{
Is osteoarthritis a deadly disease?
}

\section{Short communication}

Osteoarthritis is the most common chronic musculoskeletal condition occurring worldwide and in the United States, affecting 1 in 5 adults. ${ }^{1}$ It is a progressive condition for which there are very limited treatments and the vast majority of those are targeted toward management of pain. Osteoarthritis $(\mathrm{OA})$ risk factors include obesity, joint trauma, increasing age, female gender and reduced muscle strength to name a few. As a chronic medical disease, OA shares characteristics with other chronic medical problems inasmuch as it cannot be "cured" per se, but often times can be successfully managed. The management end-point of osteoarthritis of the hip or knee would be a total joint arthroplasty which can reduce pain and improve function. However, are there other consequences or effects of osteoarthritis that bear mention, particularly that of mortality risk? The inflammatory arthritides, particularly rheumatoid arthritis, have been associated with earlier mortality risk. ${ }^{2}$ Osteoarthritis, while not classified as an inflammatory arthritis, does have intermittent joint inflammation as a characteristic of the disease process. ${ }^{3}$ In line with the evidence of early mortality risk and rheumatoid arthritis, OA of the hip or knee has also been convincingly associated with early mortality risk in diverse populations studied.

Several large population-based cohort studies have evaluated the risk of mortality with OA of the hip or knee. Nuesch et al. ${ }^{4-7}$ examined a cohort of 1163 subjects aged $\geq 35$ years from general practices in England with both radiographic changes and symptoms of either hip or knee OA. ${ }^{4}$ Compared to the age and gender specific mortality data from England and Wales, this cohort demonstrated a higher all-cause mortality (SMR 1.55[95\% CI 1.41.-1.70]), as well as an increased cardiovascular mortality (SMR $1.71[95 \%$ CI $1.49-1.98]) .{ }^{4}$ Subsequently, data from the Study of Osteoporotic Fractures, which is a US based cohort, evaluated the mortality risk among women $\geq 65$ years of age with radiographic hip OA. ${ }^{5}$ Cause of death and mortality in this cohort was determined through use of death certificates and hospital discharge summaries. Controlling for age, BMI, education, smoking status, health status, diabetes mellitus, and stroke; women with radiographic hip OA were found to have an increased risk of all-cause mortality (HR 1.14[95\% CI 1.05-1.24]) and cardiovascular mortality (HR $1.24\left[95 \%\right.$ CI 1.09-1.41]). ${ }^{5}$

The Chingford Cohort Study evaluated a community-based population of women aged 45-64 years who resided in Northern London. ${ }^{6}$ This population included 821 women with knee OA and found that women with symptomatic knee OA had an increased all-cause mortality (HR 1.97[95\% CI 1.23-3.17]), and an increased cardiovascular mortality (HR 3.57[95\% CI 1.53-8.34]). ${ }^{6}$ While these Euro-American studies might suggest that there are several cultural or societal confounders to these findings, data from the Wuchuan Osteoarthritis Study suggests otherwise. Liu et al. examined data from 1025 residents' ages $\geq 50$ years in the Wuchuan province of China. ${ }^{7}$ They categorized knee OA as symptomatic knee pain with or without radiographic changes. Correcting for multiple confounding variables, they found that those with knee pain and radiographic changes of knee OA had increased all-cause mortality (HR 1.9[95\% CI 1.0-3.5]). ${ }^{7}$

To further support these findings there have been some recently published large scale reviews of the data. Veronese et al. ${ }^{8-10}$ performed
Volume 3 Issue I - 2018

\author{
Joseph Garry \\ Department of Family Medicine \& Community Health, \\ University of Minnesota, USA
}

Correspondence: Joseph Garry, Associate Professor, Department of Family Medicine \& Community Health, University of Minnesota, USA, Tel (6I2) 626-2338, Fax (6I2) 676-4057,Email Garry036@umn.edu

Received: December 15, 2017 | Published: January 02, 2018

a meta-analysis of the literature and found that hip or knee OA was associated with increased mortality (HR 1.18[95\% CI 1.08-1.28]). ${ }^{8}$ This study also found that hip or knee OA was associated with a $21 \%$ increase in cardiovascular mortality. Leyland et al. has presented data utilizing an individualized patient-level meta-analysis of 9889 persons from six population based cohort studies, as well as a similar review of 36,884 persons from nine cohorts ${ }^{9,10}$ The latter study was presented in the Osteoarthritis Research Society International's white paper to the US Food and Drug Administration in 2016. ${ }^{10}$ In each of these studies, subjects with symptomatic and radiographic knee OA were found to have an increased overall mortality ranging narrowly from $19-23 \%$. In the latter study, it was also demonstrated that those with symptomatic hip OA had a $20 \%$ increased mortality as compared to those without OA[95\% CI 1.04-1.37]. ${ }^{10}$

These findings of increased mortality risk associated with hip or knee osteoarthritis might suggest that an earlier approach to arthroplasty might be prudent. The data regarding this is sparse, yet a few studies have suggested otherwise. Data from the Swedish Knee Arthroplasty Register found a reduction in overall mortality after total knee arthroplasty. However, this persisted only for 12 years after which there was a sharp rise in overall mortality among OA subjects. ${ }^{11}$ For those undergoing a total hip arthroplasty, there have been findings of reduced mortality in the 5 years post-arthroplasty. ${ }^{12,13}$ However, among those patients who underwent total hip arthroplasty at ages $<50$ years, and ages 50-59 years there were increased overall mortalities (SMR 2.50[95\% CI 1.99-3.14] and 1.16[95\% CI 1.011.32] respectively). ${ }^{12}$ It also appears that beyond 5 years post-total hip arthroplasty the mortality benefit may be lost at least among the Medicare population. ${ }^{13}$ As such, while total joint arthroplasty may improve pain and function due to osteoarthritis, it may have only shorter-term benefits in abatement of the underlying mortality risk.

Osteoarthritis is the most common musculoskeletal condition in the US. The findings of both increased mortality, and increased cardiovascular mortality associated with this condition certainly suggest that we undertake further research in the management of this chronic medical condition. It appears that joint arthroplasty may simply be another modifying treatment of this disease process providing short-term mortality benefits for older patients. The benefit of earlier detection of lower extremity OA is unknown in terms of effect on mortality. Extrapolation from other work in OA disease progression suggests that earlier detection would allow clinicians more time to affect behavior change with regards to body habitus, physical activity patterns and muscle strength. However, the real 
reduction in this mortality risk will be in the understanding of $\mathrm{OA}$ onset and research into disease prevention, which translates into reductions in risk factors, as well as research into prevention of OA in the post-traumatic joint. In the interim, it is now becoming clear that clinicians need to address mortality risk with their lower extremity OA patients.

\section{Acknowledgements}

None.

\section{Conflict of interest}

The author declares no conflict of interest.

\section{References}

1. Helmick CG, Felson DT, Lawrence RC, et al. Estimates of the prevalence of arthritis and other rheumatic conditions in the United States: Part I. Arthritis Rheum. 2008;58(1):15-25.

2. Avina ZJA, Choi HK, Sadatsafavi M, et al. Risk of cardiovascular mortality in patients with rheumatoid arthritis: A meta-analysis of observational studies. Arthritis Rheum. 2008;59(12):1690-1697.

3. Kapoor M, Martel PJ, Lajeunesse D, et al. Role of proinflammatory cytokines in the pathophysiology of osteoarthritis. Nature Rev Rheumatology. 2011;7(1):33-42.

4. Nuesch E, Dieppe P, Reichenbach S, et al. All cause and disease specific mortality in patients with knee or hip osteoarthritis: population based cohort study. BMJ. 2011;342:1-8.
5. Barbour KE, Lui LY, Nevitt MC, et al. Hip osteoarthritis and the risk of all-cause and disease-specific mortality in older women: A populationbased cohort study. Arth Rheumatol. 2015;67(7):1798-1805.

6. Kluzek S, Sanchez-Santos MT, Leyland KM, et al. Painful knee but not hand osteoarthritis is an independent predictor of mortality over 23 years follow-up of a population-based cohort of middle-aged women. Ann Rheum Dis. 2015;1-8.

7. Liu Q, Niu J, Huang J, et al. Knee osteoarthritis and all-cause mortality: the wuchuan osteoarthritis study. Osteoarth Cartil. 2015;23(7):1154 1157.

8. Veronese N, Cereda E, Maggi S, et al. Osteoarthritis and mortality: A prospective cohort study and systematic review with meta-analysis. Seminars Arthritis Rheum. 2016;46(2):160-167.

9. Leyland KM, Gates LS, Sanchez SMT, et al. Knee Osteoarthritis and the Risk of Premature Mortality in the Community: An International Individual Patient Level Meta-Analysis in 9889 Subjects. Osteoarth Cartil. 2017;25(1):S29-30.

10. Osteoarthritis research society international osteoarthritis: A Serious disease. US Food and Drug Administration. USA; 2016. p. 1-103.

11. Robertsson O, Stefandsdottir A, Lidgren L, et al. Increased long-term mortality in patients less than 55 years old who have undergone knee replacement for osteoarthritis. J Bone Joint Surg Br. 2007;89(5):599603 .

12. Lie SA, Engesaeter LB, Havelin LI, et al. Mortality after total hip replacement. Acta Orthop Scand. 2000;71(1):19-27.

13. Barrett J, Losina E, Baron JA, et al. Survival following total hip replacement. JBJS (Am). 2005;87(9):1965-1971. 